\title{
Interferon gamma for diagnosing tuberculous pleural effusions
}

Somkiat Wongtim, Udomsak Silachamroon, Kiat Ruxrungtham, Visit Udompanich, Sakchai Limthongkul, Pradit Charoenlap, Chaivej Nuchprayoon

\begin{abstract}
Background-A study was undertaken to evaluate the diagnostic value of pleural fluid concentrations of interferon gamma $(\mathrm{IFN}-\gamma)$ as a marker of tuberculosis.

Methods-Patients admitted to King Chulalongkorn Memorial Hospital between April 1997 and January 1998 with a lymphocytic exudative pleural effusion were enrolled into the study. The pleural fluids were examined for cytology, staining for acid fast bacilli, and mycobacterial culture. Pathological examination and mycobacterial culture were performed on each pleural biopsy specimen. The diagnosis of tuberculosis was made when one of the following criteria was met: (1) Mycobacterium tuberculosis was isolated from either the pleural fluid or pleural tissue; (2) granulomas were demonstrated in the pleural tissue which stained positive for acid fast bacilli (AFB); or (3) in the presence of granulomas negative on staining for AFB in pleural tissue there was a response to antituberculous treatment on follow up. All pleural fluid samples were stored at $-70^{\circ} \mathrm{C}$ and the IFN- $\gamma$ level was measured by immunoassay. Analysis was made using sensitivity, specificity, and likelihood ratio for a positive test result. The best cut off point was determined by the highest likelihood ratio and receiver operating characteristic curve.
\end{abstract}

Results-A total of 66 patients were enrolled and tuberculosis was confirmed in 39 of them. The diagnoses in the nontuberculous group included malignancy (15), paramalignancy (11), and chronic pleuritis secondary to infective endocarditis (1). The mean (SE) IFN- $\gamma$ level in the pleural fluid was significantly higher in the tuberculous group than in the nontuberculous group (1493.3 (131.3) $\mathrm{pg} / \mathrm{ml}$ versus $80.1(50.4) \mathrm{pg} / \mathrm{ml}, \mathrm{p}<0.001)$. The overlap between the two groups was minimal. At the cut off value of $240 \mathrm{pg} / \mathrm{ml}$ the sensitivity was $94.9 \%$ (95\% CI 86.6 to 100$)$, the specificity was $96.3 \%$ (95\% CI 89.2 to 100 ), and the likelihood ratio for a positive test result was 25.6 .

Conclusions-The pleural fluid concentration of IFN- $\gamma$ is a good and useful diagnostic marker of tuberculosis presenting as a lymphocytic exudative pleural effusion. (Thorax 1999;54:921-924)

Keywords: interferon gamma; tuberculosis; pleural effusion
Tuberculosis is the major cause of pleural effusions in areas of high tuberculosis prevalence. ${ }^{1}$ Tuberculous pleuritis usually manifests as lymphocytic exudative pleural effusions. ${ }^{2}$ The diagnosis is commonly made by the presence of granulomas in the pleural tissue or a positive culture of Mycobacterium tuberculosis in the pleural tissue or pleural fluid. ${ }^{2}$ The yield of these methods is limited even when all tests are combined $^{13-7}$ and diagnostic difficulty still exists in some cases. Repeating the procedure may yield a positive result but it places the patient at risk of more complications and increases the cost. Many biological markers have been proposed to aid the diagnostic sensitivity. ${ }^{1}{ }^{7-9}$ Among them, interferon $\gamma$ (IFN$\gamma)$, a cytokine produced by activated CD $4+\mathrm{T}$ cells in the Th1 subset which plays an important part in cell mediated immunity and the development of tuberculous pleural effusions, has been reported to have high sensitivity and specificity. ${ }^{8-10}$ Interferon $\gamma$ has been studied in various clinical situations in which pleural effusions are present. However, it has not been investigated prospectively in patients presenting with the lymphocytic exudative pleural effusion typically seen in tuberculosis.

A study was undertaken to evaluate the diagnostic value of pleural fluid levels of IFN- $\gamma$ in the diagnosis of tuberculosis in patients presenting with lymphocytic exudative pleural effusions. Its best cut off point was obtained by the analysis of sensitivity, specificity, likelihood ratio for a positive test result, and receiver operating characteristic curve. These parameters are less likely to be affected by the prevalence of the disease in the study population. ${ }^{11}$

\section{Methods}

PATIENTS

King Chulalongkorn Memorial Hospital is a university hospital with 2000 beds. About 300 patients present annually to the Chest Unit for evaluation of pleural effusions and approximately $60 \%$ of these effusions are found to be caused by tuberculosis.

New patients with lymphocytic exudative pleural effusion were prospectively enrolled from 30 April 1997 to 20 January 1998. We defined lymphocytic exudative pleural effusion as an exudative pleural effusion ${ }^{12}$ with more than $50 \%$ lymphocytes on differential counting. ${ }^{213}$ All patients were aged 15 years or more, had significant pleural fluid detectable on physical examination, and a standard chest radiograph without contraindication for thoracentesis. Patients who had either taken antitu-

\author{
8 June 1999 \\ Accepted for publication \\ 24 June 1999 \\ Received 6 January 1999 \\ Returned to author \\ 22 March 1999
}


berculous drugs prior to thoracentesis or had previously had the cause of their effusion diagnosed were excluded. The sample size was calculated with the expectation that the test would have the sensitivity of at least $90 \%$ with no more than $10 \%$ error. The prevalence of tuberculosis was estimated at $60 \%$ among patients with lymphocytic exudative pleural effusion. At least 60 cases were required to show statistical power. The study protocol was approved by our institutional review board and written consent was obtained from all of the patients.

SPECIMEN COLLECTION AND PROCESSING

For each subject $40 \mathrm{ml}$ of pleural fluid was collected in a heparinised syringe during thoracentesis. The first $20 \mathrm{ml}$ sample was submitted for total and differential cell counting, acid fast staining, cytological examination, and measurement of protein and lactate dehydrogenase concentrations. Another $20 \mathrm{ml}$ sample was centrifuged at $3000 \mathrm{rpm}$ for 15 minutes. The supernatant was frozen at $-70^{\circ} \mathrm{C}$ within one hour after thoracentesis pending IFN- $\gamma$ measurement. The sediment was used for mycobacterial culture. Pleural biopsy specimens were taken using an Abram or Cope needle to obtain at least four pieces of tissue, three were for pathological examination and one for mycobacterial culture.

For mycobacterial culture each sample was inoculated on Ogawa medium in duplicate and assessed weekly for mycobacterial growth for a total of eight weeks. $M$ tuberculosis was identified by a positive niacin test.

IFN- $\gamma$ concentrations were measured using a commercial ELISA kit (Predicta IFN- $\gamma$ ELISA Kit, Genzyme Corp, USA) which has the sensitivity to detect concentrations of IFN- $\gamma$ as low as $3 \mathrm{pg} / \mathrm{ml}$. All samples were thawed and measured simultaneously in duplicate by a technician in a blinded fashion. Each set of samples was measured and compared with the standard controls supplied by the manufacturer. The cost of the test was approximately US $\$ 20$ per sample.

\section{DIAGNOSIS OF TUBERCULOSIS}

Patients were diagnosed as having tuberculosis when they met one of the following criteria: (1) $M$ tuberculosis was isolated from either the pleural fluid or pleural tissue; (2) granulomas were found in the pleural tissue and stained positive for acid fast bacilli (AFB); or (3) in cases of granulomas in the pleural tissue that did not stain positive for AFB there was a response to antituberculous treatment on follow up.

Malignant pleural effusions were diagnosed either by a positive pleural fluid cytology result or positive malignant cells in pleural biopsy specimens. ${ }^{14}$

The patients suspected of having malignancy on clinical grounds with initial negative results were further investigated by repeating the thoracentesis, pleural biopsy, and evaluation for primary tumour. Patients in whom a primary tumour responsible for the development of the pleural effusion was confirmed without tumour cells being identified in the pleural cavity were classified as having paramalignant effusions.

\section{STATISTICAL ANALYSIS}

The patients were divided into two groups according to the cause of their effusiontuberculous and non-tuberculous. The difference in the mean IFN- $\gamma$ levels was determined by the unpaired $t$ test. The sensitivity, specificity, and likelihood ratio for positive test result $(\mathrm{LR}+)$ of all probable cut off points were calculated as follows:

Sensitivity $=$ true positive/(true positive + false negative)

Specificity $=$ true negative/(true negative + false positive)

Likelihood ratio for positive test result $(\mathrm{LR}+)=$ sensitivity $/(1$ - specificity $)$.

The $95 \%$ confidence intervals (95\% CI) of the sensitivity or specificity were calculated from sensitivity or specificity $\pm 1.96 \mathrm{SE}$. The final analysis was made by the receiver operating characteristic (ROC) curve. The best cut off point was determined by the highest LR+ and analysis by ROC curve.

\section{Results}

During the period of recruitment 180 patients underwent thoracentesis at our unit. One hundred and nine patients were eligible for the study (untreated lymphocytic exudative pleural effusion of unknown cause) but 21 cases were excluded because of inadequate specimen collection or inappropriate handling and 22 cases were excluded because a final diagnosis could not be made, leaving 66 cases to be enrolled in the study.

The tuberculous group consisted of 39 patients of mean (SE) age 40.6 (2.7) years (range 15-75) with a male to female ratio of 5.5:1. The predisposing factors for tuberculosis were HIV infection (11 cases), diabetes mellitus ( 3 cases), and administration of steroid ( 1 case).

The non-tuberculous group consisted of 27 patients of mean (SE) age 57.4 (3.5) years (range 18-94) with a male to female ratio of 1:3.5. Fifteen had a malignant pleural effusion, 11 a paramalignant effusion, and one had chronic pleuritis secondary to infective endocarditis.

The yields of the various methods for diagnosing tuberculosis are shown in table 1 . Acid fast staining of pleural fluid was negative in all cases. The cultures of pleural fluid and pleural biopsy specimens were positive in $28.2 \%$ and $39.5 \%$ of patients, respectively. Granulomas were detected in the pleural tissue in $84.6 \%$ of patients.

Table 1 Yields of various methods for diagnosing tuberculous pleural effusion

\begin{tabular}{ll}
\hline Methods & Yields (\%) \\
\hline Pleural biopsy culture & 39.5 \\
Pleural fluid culture & 28.2 \\
Positive granuloma in pleural biopsy & 84.6 \\
Positive AFB in pleural biopsy & 43.6 \\
Positive AFB in pleural fluid & 0
\end{tabular}

Positive AFB in pleural fluid

$\mathrm{AFB}=$ acid fast bacilli. 


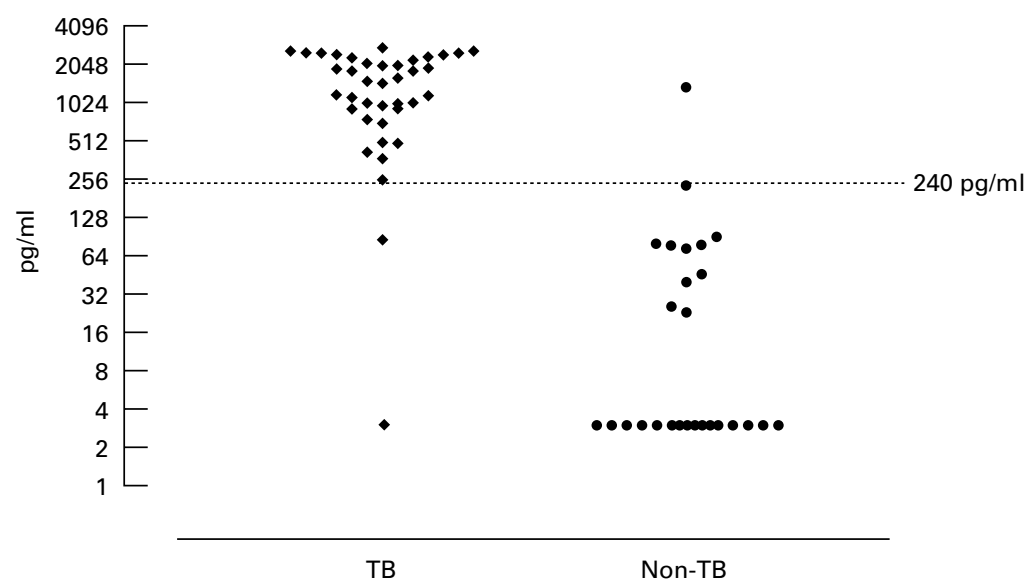

Figure 1 Distribution of interferon $\gamma$ in pleural fluid of patients with and without tuberculosis.

The mean (SE) concentration of IFN- $\gamma$ in the pleural fluid of the tuberculous group was 1493.3 (131.3) $\mathrm{pg} / \mathrm{ml}$ (range 3-2750) and of the non-tuberculous group was 80.1 (50.4) pg/ $\mathrm{ml}$ (range 3-1365). This difference was statistically significant $(\mathrm{p}<0.001)$. There was minimal overlap in the IFN- $\gamma$ levels between the two groups as shown in fig 1 .

There were two patients with a tuberculous pleural effusion who had low IFN- $\gamma$ levels $(<240 \mathrm{pg} / \mathrm{ml})$. Both were over 60 years of age and one had poorly controlled diabetes mellitus. Serological examination for anti-HIV was negative and both had non-specific findings on pleural fluid cytology and pleural biopsy. The diagnosis was confirmed by positive culture of $M$ tuberculosis from the pleural tissue and fluid. They showed an initial response to antituber-

Table 2 Sensitivity, specificity, and likelihood ratio for positive test result (LR+) for all possible cut off points

\begin{tabular}{clll}
\hline $\begin{array}{l}\text { Cut off point } \\
(p g / m l)\end{array}$ & Sensitivity & Specificity & LR+ \\
\hline 0 & 1.000 & 0.000 & 1.00 \\
22 & 0.974 & 0.593 & 2.39 \\
83 & 0.974 & 0.889 & 8.77 \\
88 & 0.949 & 0.889 & 8.54 \\
240 & 0.949 & 0.963 & 25.62 \\
1300 & 0.538 & 0.963 & 14.54 \\
1400 & 0.538 & 1.000 & - \\
2750 & 0.000 & 1.000 & - \\
\hline
\end{tabular}

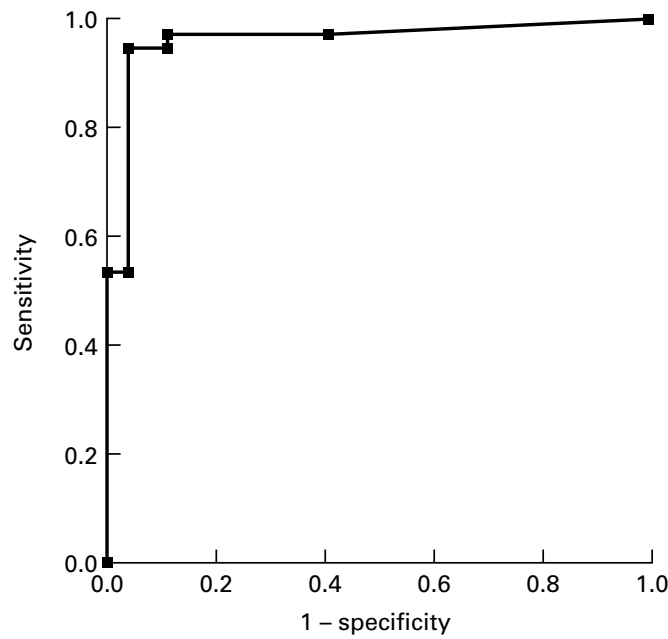

Figure 2 Receiver operating characteristic curve for using interferon $\gamma$ for diagnosis of tuberculous pleural effusions. culous treatment but did not complete the follow up.

One patient with a malignant pleural effusion had a very high level of IFN- $\gamma(1365.1 \mathrm{pg}$ / $\mathrm{ml}$ ). She was 32 years old and had a moderate right pleural effusion. Thoracentesis was performed twice and adenocarcinoma was revealed by cytological examination of the pleural fluid and pleural biopsy specimens. All specimens sent for mycobacterial culture were negative. She died soon after the diagnosis was made and no necropsy was performed.

No correlation was found between the IFN- $\gamma$ level and protein content of the pleural fluid $(r$ $=-0.23, \mathrm{p}=0.15)$. There was no difference in the mean IFN- $\gamma$ level when the groups with small, moderate, and large pleural effusions were compared (one way ANOVA, $\mathrm{p}=0.49$ ).

The HIV status was known in 16 patients in the tuberculous group (the serology for antiHIV was only done with the patient's permission). Eleven patients with positive results had a median IFN- $\gamma$ level of $1177 \mathrm{pg} / \mathrm{ml}$. The median of the five remaining cases was 503 pg/ $\mathrm{ml}$. This difference was not statistically significant by the median test $(\mathrm{p}=0.128)$.

All possible cut off points were determined and sensitivity, specificity, and LR+ for each cut off were calculated and are shown in table 2 . An ROC curve based on these data was constructed as shown in fig 2 .

\section{Discussion}

Tuberculous pleural effusions are thought to result from rupture of a subpleural caseous focus in the lung into the pleural space. ${ }^{4}$ Delayed type hypersensitivity is induced by tuberculoprotein and leads to the development of a pleural effusion. Lymphocytes from this pleural fluid produce high concentrations of IFN- $\gamma$ after in vitro stimulation with PPD. ${ }^{15-17}$ High levels of IFN- $\gamma$ are found in patients with tuberculous pleural effusions, while it is almost undetectable in pleural effusions of other aetiologies. ${ }^{18-20}$

The level of IFN- $\gamma$ in the pleural fluid has been reported to have a high sensitivity and specificity in tuberculous pleural effusion. ${ }^{8-10}$ However, most of the earlier studies were conducted in various types of pleural effusion including patients who were unlikely to have tuberculosis. Our study was carried out prospectively in a clinical situation that would be used in practice. The results reveal that, in lymphocytic exudative pleural effusion, IFN- $\gamma$ levels are significantly higher in tuberculous than in non-tuberculous disease. The bimodal distribution and minimal overlap between the two groups makes it possible to differentiate tuberculosis from nontuberculous disease with a high sensitivity, specificity, and the likelihood ratio for a positive test result. Analysis of the data using a ROC curve (fig 2) shows the curve above the line $x=$ $y$ and markedly angulated at $240 \mathrm{pg} / \mathrm{ml} \mathrm{IFN}-\gamma$. According to these data the best cut off point is therefore $240 \mathrm{pg} / \mathrm{ml}$. This yields a sensitivity of $94.9 \%$ (95\% CI 86.6 to 100$)$, a specificity of $96.3 \%$ (95\% CI 89.2 to 100 ), and LR+ of 25.6 . As previously discussed, a test in which the LR+ 
is greater than 10 is a very useful diagnostic tool because it generates a large and often conclusive change from pretest to post-test probability. ${ }^{21}$

Our cut off point differs from that found in previous studies. However, all the previous reported cut off points differ from each other. This might be due to different mode sample collection and processing methods, and/or different methods of measuring IFN $-\gamma$. We suggest that the users should develop their own cut off point if the methods they use are different from ours.

The prevalence of tuberculosis in this study was $59.1 \%$, higher than in others previously published. The prevalence of tuberculosis in this region is high and is related to the AIDS epidemic. Nevertheless, the sensitivity, specificity, and likelihood ratio of the test are independent of the high prevalence of tuberculosis. ${ }^{11}$ The positive predictive value (PPV) and negative predictive value (NPV) can be calculated if the prevalence of tuberculosis in the population is known. ${ }^{9}$

Pleural fluid levels of IFN- $\gamma$ were found to be high in tuberculosis patients with immunosuppression. ${ }^{10}$ In this study there were no differences in the values between HIV and non-HIV patients. Similar results were reported by Villena et $a l^{10}$ although the number of HIV+ patients in both studies was rather small. There was one case of chronic steroid usage in whom the level of IFN- $\gamma$ was higher than the proposed cut off point. Neverthelesss, the data do not indicate a difference in IFN- $\gamma$ level between immunocompetent and immunosuppressed patients.

A few cases with false positive and false negative results were reported by Valdes and colleagues ${ }^{9}$, Villena and colleagues, ${ }^{10}$ and in this study. No consistent explanation can be made for the finding that IFN- $\gamma$ was very low (or even undetectable) in some patients with tuberculosis and was high in some cases of non-tuberculosis. Valdes and colleagues suggested that low levels of IFN- $\gamma$ might occur in small tuberculous effusions but this was not found by us or in other studies. ${ }^{10}$

Our yields for a positive diagnosis according to standard procedures for tuberculosis were low, as has been reported previously. ${ }^{13-7}$ Studies using the newly developed technique of mycobacterial culture in patients with tuberculous pleural effusions reported only marginal improvement. $^{722}$ Of the standard methods, pleural biopsy had the highest yield $(84.6 \%)$ which was, however, lower than the use of pleural fluid IFN- $\gamma$ level $(96.3 \%)$.

In conclusion, an increase in the IFN- $\gamma$ level in the pleural fluid is a good and useful diagnostic marker of pleural tuberculosis presenting as a lymphocytic exudative pleural effusion. Further investigations are required to clarify the characteristics of false positive and false negative cases. However, the issues of cost effectiveness, standardisation, and quality control have to be taken into consideration for further clinical implications, particularly in the developing world.

The authors thank the staff of the Chest Unit and Department of Pathology and Clinical Pathology for specimen collection and processing, Mr Somboon Nookai for measurement of IFN- $\gamma$, and Drs Sven-Iver Lorenzen and Chris Duncombe for their critical comments. This study was supported in part by the Ratchadapisek Sompotch Fund.

1 Promkiamon B. Comparison of various diagnostic tests in tuberculous pleuritis. Thoracic Society of Thailand Newsletter 1997;6:5.

2 Light RW. Pleural diseases. 3rd ed. Baltimore: William \& Wilkins, 1995.

3 Levine H, Metzger W, Lacera D, et al. Diagnosis of tuberculous pleurisy by culture of pleural biopsy specimens. Arch Intern Med 1970;126:269-71.

4 Berger HW, Mejia E. Tuberculous pleurisy. Chest 1973;63: $88-92$.

5 Scharer L, McClement JH. Isolation of tubercle bacilli from needle biopsy specimens of parietal pleura. Am Rev Respir Dis 1968:97:466 8.

6 Epstein DM, Kline LR, Albelda SM, et al. Tuberculous pleural effusions. Chest 1987;91:106-9.

7 Maartens G, Bateman ED. Tuberculous pleural effusions: increased culture yield with bedside inoculation of pleural fluid and poor diagnostic value of adenosine deaminase. Thorax 1991;46:96-9.

8 Aoki Y, Katoh O, Nakanishi Y, et al. Comparison study of IFN- $\gamma$, ADA, and CA125 as the diagnostic parameters in tuberculous pleuritis. Respir Med 1994;88:139-43.

9 Valdes L, Jose ES, Alvarez D, et al. Diagnosis of tuberculous pleurisy using the biologic parameters adenosine deaminase, lysozyme, and interferon gamma. Chest 1993;103: 458-65.

10 Villena V, Lopez-Encuentra A, Echave-Sustaeta J, et al. Interferon- $\gamma$ in 338 immunocompromised and immunoInterferon- $\gamma$ in 338 immunocompromised and immunoRespir ₹ 1996;9:2639-9.

11 Metz CE. Basic principles of ROC analysis. Semin Nucl Med 1978;8:283-98

12 Light RW, MacGregor MI, Luchsinger PC, et al. Pleural effusions: the diagnostic separation of transudates and exudates. Ann Intern Med 1972;77:507-13.

13 Yam LT. Diagnostic significace of lymphocytes in pleural effusions. Ann Intern Med 1967;66:972-82.

14 Fenton KN, Richardson JD. Diagnosis and management of malignant pleural effusions. Am f Surg 1995;171:69-74.

15 Shimokata K, Kuwachi H, Kishimoto H, et al. Local cellular immunity in tuberculous pleurisy. Am Rev Respir Dis 1982; 126:822-4.

16 Shimokata K, Kishimoto H, Takagi E, et al. Determination of the T-cell subset producing gamma-interferon in tuberculous pleural effusion. Microbiol Immunol 1986;30:35361

17 Ribera E, Espanol T, Martinez-Vazquez JM, et al. Lymphocytic proliferation and gamma-interferon production after in vitro stimulation with PPD. Differences between tuberculous and non tuberculous pleurisy in patients with positive tuberculin skin test. Chest 1990;97:1381-5.

18 Barnes PF, Lu S, Abrams JS, et al. Cytokine production at the site of disease in human tuberculosis. Infect Immun 1993;61:3482-9.

19 Ribera E, Ocana I, Martinez-Vazquez JM, et al. High level of interferon gamma in tuberculous pleural effusion. Chest 1988:93:308-11.

20 Shimokata K, Saka H, Murate T, et al. Cytokine content in pleural effusion. Comparison between tuberculous and carcinomatous pleurisy. Chest 1991;99:1103-7.

21 Jaeschke R, Guyatt GH, Sackett D. Users' guides to the medical literature.III. How to use an article about a diagnostic test. B. What are the results and will they help me in caring for my patients? FAMA 1994;271:703-7.

22 Cheng AF, Li MSK, Chan CY, et al. Evaluation of three culture media and their combinations for isolation of Mycobacterium tuberculosis from pleural aspirates of patients with tuberculous pleurisy. 7 Trop Med Hyg 1994;97:24953. 